\section{DOI: $10.21105 /$ joss. 01757}

\section{Software}

- Review [ד

- Repository u

- Archive [ᄌ

Editor: Arfon Smith ¿

Reviewers:

- Otukss

- Opgrete

Submitted: 03 September 2019 Published: 21 November 2019

\section{License}

Authors of papers retain copyright and release the work under a Creative Commons Attribution 4.0 International License (CC-BY).

In partnership with

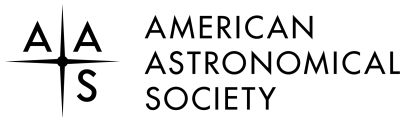

This article and software are linked with research article DOI 10.3847/1538-4357/ab4f75, published in the Astrophysical Journal.

\title{
MAESTROeX: A Massively Parallel Low Mach Number Astrophysical Solver
}

\section{Duoming Fan ${ }^{1}$, Andrew Nonaka $^{1}$, Ann Almgren ${ }^{1}$, Donald Willcox ${ }^{1}$, Alice Harpole ${ }^{2}$, and Michael Zingale ${ }^{2}$}

1 Center for Computational Sciences and Engineering, Lawrence Berkeley National Laboratory 2 Department of Physics and Astronomy, Stony Brook University

\section{Summary}

MAESTRDeX is a massively parallel, finite-volume $\mathrm{C}++/ \mathrm{F90}$ solver for low Mach number astrophysical flows. Our code utilizes a low Mach number equation set allowing for more efficient, long-time integration of highly subsonic flows compared to compressible approaches. The recommended range of applicability is for flows where the Mach number does not exceed $\sim 0.1$.

In highly subsonic astrophysical phenomena, sound waves carry sufficiently little energy that they do not significantly affect the convective dynamics of the system. In many of these flows, modeling long-time convective dynamics are of interest, and numerical approaches based on compressible hydrodynamics are intractable, even on modern supercomputers. One approach to this problem is to use low Mach number models. In our approach, asymptotic model equations are employed that do not contain sound waves. Our customized low Mach number model retains compressibilitiy effects due to, e.g., nuclear energy release, large-scale atmospheric stratification, compositional changes, and thermal diffusion. When the Mach number (the ratio of the characteristic fluid velocity over the characteristic sound speed; $\mathrm{Ma}=\mathrm{U} / \mathrm{c}$ ) is small, the resulting system can be numerically integrated with much larger time steps than a compressible model, i.e., at least a factor of $1 /$ Ma larger. Furthermore, MAESTROeX provides a significant advantage over anelastic approaches in that our model can robustly handle large perturbations in density and temperature. For a more detailed comparison to other astrophysical approaches to low Mach number flow, see (Fan, Nonaka, Almgren, Harpole, \& Zingale, 2019).

MAESTROeX is suitable for modeling full spherical stars as well as planar simulations of dynamics within localized regions of a star, and can robustly handle several orders of magnitude of density and pressure stratification. The code leverages the new AMReX software framework (Zhang et al., 2019) for block-structured adaptive mesh refinement (AMR) applications, allowing for scalability to large fractions of leadership-class machines. Our approach to parallization uses a hybrid MPI/OpenMP approach, with increasing GPU support. Microphysics are provided by the Starkiller-Astro libraries (StarKiller Microphysics Development Team et al., 2019). The code contains documentation through sphinx, a large suite of test problems, and nightly regression tests on a number of architectures.

Since the series of papers on its predecessor MAESTRO have been published (see (Nonaka et al., 2010) and references therein), numerous developments have been made to MAESTRDeX to reduce algorithm complexity and improve parallel performance. The current numerical algorithm (Fan et al., 2019) couples modules for advection (corner transport upwind), reactions (VODE), thermal diffusion (linear solvers / multigrid), pressure-projection approaches (linear solvers / multigrid), and spatial mapping routines used to define and evolve a one-dimensional hydrostatic base state. MAESTROeX has been used to study a number of problems including 
convection in Chandrasekhar mass models for type la supernovae, convection in massive stars, sub-Chandrasekhar white dwarfs, and type I X-ray bursts.

\section{Acknowledgements}

The work at LBNL was supported by the U.S. Department of Energy's Scientific Discovery Through Advanced Computing (SciDAC) program under contract No. DE-AC02-05CH11231. The work at Stony Brook was supported by DOE/Office of Nuclear Physics grant DE-FG0287ER40317 and through the SciDAC program DOE grant DE-SC0017955. We also acknowledge contributors to the previous, pure F90 implementation of MAESTRO, John Bell (LBL), Chris Malone (LANL), and Michael Lijewski (LBL).

\section{References}

Fan, D., Nonaka, A., Almgren, A. S., Harpole, A., \& Zingale, M. (2019). MAESTROeX: A massively parallel low mach number astrophysical solver. The Astrophysical Journal, 887(2). doi:10.3847/1538-4357/ab4f75

Nonaka, A., Almgren, A. S., Bell, J. B., Lijewski, M. J., Malone, C. M., \& Zingale, M. (2010). MAESTRO: An adaptive low mach number hydrodynamics algorithm for stellar flows. The Astrophysical Journal Supplement Series, 188(2), 358. doi:10.1088/0067-0049/188/2/358

StarKiller Microphysics Development Team, Bishop, A., Fields, C. E., Jacobs, A. M., Katz, M., Li, X., Malone, C., et al. (2019, May). Starkiller-astro/microphysics: Microphysics 19.05. doi:10.5281/zenodo. 2656476

Zhang, W., Almgren, A., Beckner, V., Bell, J., Blaschke, J., Chan, C., Day, M., et al. (2019). AMReX: A framework for block-structured adaptive mesh refinement. Journal of Open Source Software, 4(37), 1370. doi:10.21105/joss.01370 